\title{
Automated Image Analysis of HER2 Fluorescence In Situ Hybridization to Refine Definitions of Genetic Heterogeneity in Breast Cancer Tissue
}

\author{
Gedmante Radziuviene, ${ }^{1,2}$ Allan Rasmusson, ${ }^{1}$ Renaldas Augulis, ${ }^{1,2,3}$ \\ Daiva Lesciute-Krilaviciene, ${ }^{1,2}$ Aida Laurinaviciene, ${ }^{1,3}$ Eduard Clim, ${ }^{4}$ \\ and Arvydas Laurinavicius ${ }^{1,3}$ \\ ${ }^{1}$ National Center of Pathology, Affiliate of Vilnius University Hospital Santariskiu Clinics, P. Baublio 5, LT-08406 Vilnius, Lithuania \\ ${ }^{2}$ Faculty of Natural Sciences, Vilnius University, M. K. Ciurlionio 27, LT-03103 Vilnius, Lithuania \\ ${ }^{3}$ Faculty of Medicine, Vilnius University, M. K. Ciurlionio 21, LT-03101 Vilnius, Lithuania \\ ${ }^{4}$ TissueGnostics, Vienna, Austria \\ Correspondence should be addressed to Arvydas Laurinavicius; arvydas.laurinavicius@vpc.lt
}

Received 19 December 2016; Revised 20 March 2017; Accepted 26 April 2017; Published 28 May 2017

Academic Editor: Hirotaka Iwase

Copyright (C) 2017 Gedmante Radziuviene et al. This is an open access article distributed under the Creative Commons Attribution License, which permits unrestricted use, distribution, and reproduction in any medium, provided the original work is properly cited.

\begin{abstract}
Human epidermal growth factor receptor 2 gene- (HER2-) targeted therapy for breast cancer relies primarily on HER2 overexpression established by immunohistochemistry (IHC) with borderline cases being further tested for amplification by fluorescence in situ hybridization (FISH). Manual interpretation of HER2 FISH is based on a limited number of cells and rather complex definitions of equivocal, polysomic, and genetically heterogeneous (GH) cases. Image analysis (IA) can extract highcapacity data and potentially improve HER2 testing in borderline cases. We investigated statistically derived indicators of HER2 heterogeneity in HER2 FISH data obtained by automated IA of 50 IHC borderline (2+) cases of invasive ductal breast carcinoma. Overall, IA significantly underestimated the conventional HER2, CEP17 counts, and HER2/CEP17 ratio; however, it collected more amplified cells in some cases below the lower limit of GH definition by manual procedure. Indicators for amplification, polysomy, and bimodality were extracted by factor analysis and allowed clustering of the tumors into amplified, nonamplified, and equivocal/polysomy categories. The bimodality indicator provided independent cell diversity characteristics for all clusters. Tumors classified as bimodal only partially coincided with the conventional GH heterogeneity category. We conclude that automated high-capacity nonselective tumor cell assay can generate evidence-based HER2 intratumor heterogeneity indicators to refine GH definitions.
\end{abstract}

\section{Introduction}

Amplification and/or overexpression of the human epidermal growth factor receptor 2 (HER2) oncogene is observed in approximately $20 \%$ of invasive breast tumors and is associated with worse prognosis and need for targeted therapy [1-3]. Accurate and precise detection of HER2 status is thus essential for individual therapy decision for patients with breast cancer $[4,5]$. The most common procedure for determining HER2 status in breast carcinoma is based on immunohistochemistry (IHC) evaluation of HER2 expression followed by HER2 amplification test in IHC borderline (2+) cases. HER2 amplification is commonly tested by fluorescence in situ hybridization (FISH) detection of HER2 and chromosome 17 (CEP17) FISH signals [6]. Current ASCO/CAP 2013 guidelines [3] state that HER2 amplification must be determined from manual counts of discrete HER2 and CEP17 signals in 40 nuclei per case with an additional 20 nuclei in equivocal cases.

While the majority of HER2 positive and negative tumors can be readily be identified by this procedure, analysis of borderline and heterogeneous tumors may be hampered by cellto-cell diversity regarding HER2 copy number. HER2 amplified cells may be clustered in specific areas or scattered and 
intermixed with nonamplified cells $[7,8]$. CEP17 polysomy poses another dilemma in interpreting HER2 FISH results; in addition, an increased number of CEP17 signals are related to amplification of the centromeric region rather than to true polysomy $[9,10]$. The interpretation of the diversity of cells is further complicated by possible influence of both technical aspects and observer subjectivity in selecting countable nuclei [11-13].

To address the issue of diversity, HER2 genetic heterogeneity $(\mathrm{GH})$ was defined by the College of American Pathologists (CAP) as the presence of more than $5 \%$ but less than $50 \%$ of infiltrating tumor cells with a HER $2 /$ CEP17 ratio $>2.2$ when using a control probe (or $>6$ HER2 signals per cell when using a probe for HER2 only) [14]. Seol et al. reported that disease-free survival times in patients with $\mathrm{GH}$ were significantly shorter compared with patients without GH [15]. Nevertheless, utility of the GH definition has been questioned by many studies [11-13, 16-18]. In particular, the incidence of GH was reported to vary between $11 \%$ and $40 \%$ [11], and a higher rate of GH was reported when established by HER2/CEP17 ratio compared to that established by HER2 alone (23\% and $7 \%$, resp.) [18]. Furthermore, the GH definition has been shown as noninformative of the underlying distribution within a tumor cell population since the degree of GH increased along with the overall HER2/CEP17 ratio to approach the "cut-off point" of 2.2 [13]. On a similar note, Öhlschlegel et al. reported that the GH was significantly associated with CEP17 polysomy [19].

Manual evaluation of HER2 FISH results is not only a time-consuming and somewhat tedious procedure, but, more importantly, it is based on evaluation of cell diversity in a limited sample and is prone to selection bias [11-13]. The current clinical guidelines for establishing equivocal/heterogeneous cases are complex, involving different quantities and cut-offs (signal counts, their ratio, and proportion of cells amplified) which make them hard to follow. One may argue that the manual assessment of HER2 FISH status is not yet sufficiently standardized in terms of tissue and cell sampling for counting of FISH signals. This was reflected in previous studies of automated FISH evaluation with sampling varying within 20-60 nuclei $[20,21]$ to a few TMA cores [22] or a few fields selected from breast cancer sections [23].

Automation of HER2 FISH test by means of image analysis (IA) has been proposed by commercial platforms [21-25] to reduce workload and improve precision. While still dependent on good quality samples and standardization of all procedures, IA can aid as decision support tool $[21,23,26]$. Besides the benefits of IA for computer-assisted quantification of FISH signals, significant increases in cell sampling capacity may serve for better assessment of equivocal and heterogeneous cases [20,21].

Our study explores if objective, statistically derived indicators of HER2 intratumor heterogeneity can be obtained from high-capacity data extracted by IA applied to HER2 FISH digital images.

\section{Materials and Methods}

2.1. Patients and Samples. The study included 50 female patients with invasive ductal breast carcinoma diagnosed as borderline HER2 IHC (2+), treated at the National Cancer Institute (Vilnius, Lithuania), and investigated at the National Center of Pathology (Vilnius, Lithuania) between September 2012 and February 2015. The study was approved by the Lithuanian Bioethics Committee.

2.2. Fluorescence In Situ Hybridization. $4 \mu \mathrm{m}$ thick sections were stained with PathVysion HER2 DNA Probe Kit (Abbott Molecular, Des Plaines, IL, USA). In this kit, a fluorescently labeled (SpectrumOrange) DNA probe recognizing the HER2 locus (17q11.2-q12) is used in conjunction with a fluorescently labeled (SpectrumGreen) DNA probe recognizing the centromeric region of CEP17 (17p11.1-q11.1). Tissue sections were mounted on positively charged slides, heated overnight at $56^{\circ} \mathrm{C}$, deparaffinized in xylene, dehydrated in absolute ethanol, and air-dried. The slides were placed in $0.2 \mathrm{~N} \mathrm{HCl}(\mathrm{pH} 0.24)$ for $20 \mathrm{~min}$, washed in a $2 \mathrm{x}$ SSC buffer ( $\mathrm{pH}$ 7.0), and incubated with pretreatment $1 \mathrm{~N} \mathrm{NaSCN}$ solution (from Vysis Paraffin Pretreatment Kit, Abbott Molecular, Des Plaines, IL, USA) for $30 \mathrm{~min}$ at $80^{\circ} \mathrm{C}$. Subsequently, a protease digestion was performed at $37^{\circ} \mathrm{C}$ for $26 \mathrm{~min}$. The probe mixture was applied to the target tissue and the cover slips were sealed with rubber cement. Denaturation for $5 \mathrm{~min}$ at $72^{\circ} \mathrm{C}$ following hybridization for $19 \mathrm{~h}$ at $37^{\circ} \mathrm{C}$ was performed in a hybridizer (DAKO Diagnostics, Glostrup, Denmark). After hybridization, the slides were washed in $2 \mathrm{x} \mathrm{SSC} / 0.3 \% \mathrm{NP}-40$ at $72^{\circ} \mathrm{C}$ for $2 \mathrm{~min}$, air-dried before counterstaining with 4,6-diamidino-2-phenylindole (DAPI) (Invitrogen Corporation, Carlsbad, USA), and covered with a glass coverslip.

2.3. Image Acquisition. TissueFAXS-plus (TissueGnostics, Vienna, Austria), a medical device certified for "in vitro" diagnostic (IVD) developed and produced in accordance with ISO13485, was used to scan representative regions of all samples. PanApo 63x/1.4 oil objective (Zeiss, Göttingen) was used for acquiring digital images using a PCO Pixelfly CCD camera. Single band-pass filters are fitted to record nuclei (DAPI), HER2 (Acridine), and CEP17 (FITC) in separately. Each region consisted of a minimum of 4 field of views (FOVs). Image acquisition time of each channel was adjusted to 200 milliseconds.

Digital images were stitched together to regions of interest (ROIs). Each FOV was acquired at $63 \mathrm{x}$ magnification and stored at the resolution of 1392 by 1024 pixels, yielding a pixel size of $0.16 \mu \mathrm{m}$. To ensure that all signals inside the thick tissue section are available for image analysis, images were acquired using z-stacks composed of 9 steps with a step size of $0,45 \mu \mathrm{m}$. Extended depth of focus algorithm of TissueFAXS was used to combine the multiple focal planes. The algorithm is using only the sharpest structures of each layer. An illustration is given in Supplementary Figure 1 (see Supplementary Material available online at https://doi.org/10.1155/2017/2321916). 
2.4. HER2 FISH Evaluation. Two observers evaluated the mean number of HER2 and CEP17 signals and the HER2/ CEP17 ratio per nucleus by conventional manual procedure (MP): 40 nuclei were examined in two or more fields; for equivocal cases, additional 20 nuclei were evaluated. HER2/ CEP17 ratios were calculated per tumor by total number of HER2 signals divided by total number of CEP17.

For the automated analysis, ROIs were manually selected for the scanning to ensure good representation and quality of the tumor sample. Automated segmentation of both nuclei and FISH signals was performed with StrataQuest v.205 (TissueGnostics $\mathrm{GmbH}$ ). All automatically detected nuclei, HER2, and CEP17 signals (automated data (AD)) were reviewed and edited by an observer (GR) on the digital images to produce a set of corrected data (CD) for quality assurance. All nuclei were considered (no filtering of nuclei with less than one of each signal as required by the FISH evaluation guidelines was done before the correction). Subsequently, nuclei without signals or with only one HER2 or CEP17 signal were excluded from further analysis during the statistical analysis.

HER2 amplification status was determined according to the ASCO/CAP guidelines [3] and CEP17 polysomy was defined as an average CEP17 copy number $\geq 3$ [27]. The manual analysis utilized MP data, that is, counts within 40-60 nuclei selected by the observers, while the automated analysis used $\mathrm{AD}$ data from all extracted nuclei (except the nuclei with insufficient number of FISH signals as indicated above).

The HER2 intratumoral heterogeneity was estimated by (1) the CAP 2009 guidelines, GH, which were applied to both $\mathrm{MP}$ and $\mathrm{AD}$ to compare the effect of number of included nuclei on the heterogeneity measure and (2) statistical bimodality indicators: Ashman's D and bimodality index. Briefly, bimodality indicators are functions of the parameters describing two Gaussian distributions fitted to the data. The bimodality indicators were calculated for $\mathrm{AD}$ distributions of HER2, CEP17, and HER2/CEP17 ratio as extracted per cell by IA.

2.5. Statistical Analysis. To assess the accuracy of the AD HER2 copy number, CEP17 copy number and HER2/CEP17 ratio were compared to the CD by paired $t$-test and linear regression. Note that the quality assurance comparison considered all extracted nuclei. Subsequently, the verified AD had the insufficient nuclei (as determined by the ASCO/CAP guidelines) filtered out before comparison to MP. The relationships between signals, ratios, and the bimodality indicators were investigated by factor analysis including results from both AD and MP. On the resulting factor scores, clusters were extracted by the $k$-Means method to explore potential stratification of the cases. Statistical analysis was performed with SAS 9.3 software and $R$ 3.1.2. $p$ values $<0.05$ were considered statistically significant.

\section{Results}

3.1. Comparison of Automated and Corrected HER2 FISH Data. Overall, 36,154 nuclei were detected in the digital images from 50 patients. Of those, 27,266 (75.4\%) were correctly segmented. 5,626 (15.6\%) were under- or oversegmented, and 3,262 (9.0\%) were not detected. The mean number of nuclei per tissue section was 723, ranging from 192 to 1,789 . The HER2/CEP17 ratios for automated data (AD) and corrected data (CD) were calculated according to the manual procedure (MP) (a sum of HER2 signals divided by sum of CEP17).

Overall, 87,092 HER2 and 65,309 CEP17 signals were detected by the AD. Among them, 81,704 (93.8\%) HER2 signals and 1,116 (96.6\%) CEP17 signals were correctly detected, while 2,163 (2.5\%) and 1,116 (1.7\%) were falsely detected, and $3,225(3.7 \%)$ and $1,115(1.7 \%)$ were undetected, respectively.

Paired $t$-test revealed no significant bias between the AD and CD for mean CEP17 copy number (average difference $-0.0023, \mathrm{CI}=[-0.013 ; 0.008], p=0.6614)$ and negligible bias for mean HER2 copy number (average difference 0.046, CI $=[0.013 ; 0.078], p=0.0072)$ and mean HER2/CEP17 ratio (average difference $0.025, \mathrm{CI}=[0.005 ; 0.0045], p=0.0149$ ). Linear regression analysis showed perfect agreement for all three variables determined by $\mathrm{AD}$ and $\mathrm{CD}$ (Table 1).

3.2. Comparison of Automated and Manual FISH Results. HER2 and CEP17 results obtained by MP and AD (after exclusion of the nuclei with insufficient FISH signals) were compared. Paired $t$-test revealed significantly lower values obtained by AD compared to the MP data: mean HER2 copy number (average difference $-1.428, \mathrm{CI}=[1.188 ; 1.668]$, $p<0.0001$ ), mean CEP17 copy number (average difference $-0.580, \mathrm{CI}=[0.483 ; 0.676], p<0.0001)$, and HER2/CEP17 ratio (average difference $-0.240, \mathrm{CI}=[0.150-0.330], p<$ 0.0001).

Analysis by linear regression analysis (Table 1) confirmed the underestimation bias of HER2, CEP17, and HER2/CEP17 ratio by $\mathrm{AD}$ when compared to the $\mathrm{MP}$ data. The perfect agreement between the $\mathrm{AD}$ and $\mathrm{CD}$ shows that there was no significant FISH signal loss during detection by the IA. Yet, one can question if signals could be lost due to quenching or deficiencies from scanning thin focal planes from a thick section. The latter possibility was ruled out since $z$-stacks were acquired and combined to extended focus images; see Supplementary Figure 1. In addition, quality control, comparing the FISH signals by live microscope to the corresponding signals in the scanned images, was performed without any indication of possible signal losses due to signal quenching or scanning process deficiencies.

3.3. Impact of Manual and Automated Data on Genetic Heterogeneity Expression. AD underestimated both HER2 and CEP17 counts and HER2/CEP17 ratio per case (Table 1). Similarly, Figure 1(a) shows the percentages of amplified cells were lower in AD compared to MP (Amp_Cell_\%_A and Amp_Cell_\%_M, resp.) in the range above 25\% by MP; however, Amp_Cell_\%_A were higher (reaching up to 20\%) than Amp_Cell_\%_M in the range below 5\% by MP.

Figures 1(b) and 1(c) demonstrate how the MP data adhere to the current guidelines for both amplification and GH. The tumors are stratified into nonamplified, equivocal, and amplified (Figure 1(b)); however, a clear gap occurs in 
TABLE 1: Regression analysis of HER2 copy number, CEP17 copy number, and HER2/CEP17 ratio. Automated data (AD) was tested as dependent variable to estimate its prediction from the corrected data (CD) and manual procedure (MP) results.

\begin{tabular}{|c|c|c|c|c|c|}
\hline Variable & $R 2$ & Intercept & Intercept $p$ & Slope & Slope $p$ \\
\hline \multicolumn{6}{|c|}{ AD dependent, $\mathrm{CD}$ explanatory } \\
\hline HER 2 copy number & 0.989 & 0.209 & $p<0.0001$ & 0.918 & $p<0.0001$ \\
\hline CEP17 copy number & 0.992 & 0.073 & $p=0.0106$ & 0.968 & $p<0.0001$ \\
\hline HER2/CEP17 ratio & 0.985 & 0.099 & $p=0.0002$ & 0.915 & $p<0.0001$ \\
\hline \multicolumn{6}{|c|}{ AD dependent, MP explanatory } \\
\hline HER2 copy number & 0.851 & 0.610 & $p=0.0004$ & 0.557 & $p<0.0001$ \\
\hline CEP17 copy number & 0.84 & 0.464 & $p<0.0001$ & 0.621 & $p<0.0001$ \\
\hline HER2/CEP17 ratio & 0.92 & 0.335 & $p<0.0001$ & 0.784 & $p<0.0001$ \\
\hline
\end{tabular}

the determination of GH cases (Figure 1(c)). Figure 1(d) reveals the effect of underestimation by $\mathrm{AD}$ with fewer cases being amplified or equivocal as they are downgraded into the range for negative cases. The same occurs for the distribution of amplified cell percentages (Amp_Cell_\%_A), but, importantly, a continuous distribution of amplified cell percentages can be noted for AD (Figure 1(e)).

Since the current clinical guidelines for amplification and polysomy are defined by the cut-off values for MP data, the $\mathrm{AD}$ cannot be readily used for decision support without proper validation. Nevertheless, the more continuous distributions obtained by high-capacity $\mathrm{AD}$ may provide more informative measures of cell diversity. One benefit may be related to improved detection of scattered amplified cells which could be missed by MP as it requires tedious screening of large tissue areas. To test this hypothesis, we measured the median distance between the nearest amplified nuclei (with a HER2/CEP17 ratio > 2.2) and found significantly ( $p=0.0138)$ sparser distribution of amplified nuclei in the $\mathrm{AD}$ sets of the 24 potentially heterogeneous cases compared to the 8 cases that were detected as GH by MP (see Supplementary Figure 2). The second benefit of high-capacity analysis is the opportunity to calculate proper objective bimodality indicators; here we focus on bimodality as determined by Ashman's D. To establish a minimal sample size required for reliable detection of bimodality based on Ashman's D criterion, the indicator was calculated on randomly subsampled cells from the $\mathrm{AD}$ to simulate incrementally increasing sampling size (from 40 to 1,000 nuclei). With Ashman's D calculated as the mean of 1,000 sampling iterations performed for each sample size, we found that the second peak in the distribution could rarely be detected (in less than half of the subsampling iterations) in a sample size below 200 cells in the tumors $(n=23)$ which were categorized as bimodal based on their full sample size. Accordingly, Ashman's D estimates in this group of tumors revealed improving detection of bimodality (Ashman's D > 2) with increasing sample size where 800 cells potentially would be required for robust detection of this feature (see Supplementary Figure 3). Optimal sampling requirements should be established in larger data sets; however, the simulations clearly show that the required sample size is well beyond the MP capacity.
3.4. Factor Analysis. The pattern of the 3 factors extracted is presented in Figure 2(a). Factor 1 is characterized by strong positive loadings of the variables indicative of HER2 amplification (including HER2 counts, HER2/CEP17 ratios, and percentages of amplified cells by MP and AD) and is therefore interpreted as the amplification factor. Accordingly, factor 2 can be taken as factor of polysomy. Factor 3 (Figure 2(b)) was characterized by strong positive loadings of the bimodality indicators (mainly from Ashman's D estimated from HER2 and CEP17 distributions, less from the HER2/CEP17 data) and was named the bimodality factor.

3.5. Cluster Analysis. While, by definition, the factors are linearly independent, the factor score plots (Figures 2(c)-2(e)) revealed potential nonlinear relationship between the amplification and polysomy factors and clustering of the tumors. A cluster analysis of the 3 factor scores extracted 4 rather distinct clusters presented in Figure 3 and Table 2 (see Table 1 in Supplementary Material for complete listing). The clusters 1 and 2 (containing one and nine cases, resp.) revealed variable degree of amplification and bimodality factor scores. Cluster 3 was represented by nonamplified tumors (18 cases); however, a significant proportion of them revealed high HER2 bimodality score (33.3\% cases with Ashman's D > 2). Cluster 4 consisted mainly of equivocal and polysomy cases (22 cases).

While the cluster analysis distinctly stratified the tumors into amplified, nonamplified, and equivocal/polysomy types, the bimodality factor was variable in all the clusters and provided independent characteristic for the cell diversity. Examples from different clusters are presented in Figure 4. Bimodality of HER2 and/or HER2/CEP17 distribution could be detected as an independent feature in both negative and amplified cases, as in Figures 4(a), 4(b), and 4(c), respectively (none of these cases were categorized as GH by MP). Importantly, the cluster of equivocal and polysomic cases, in Figures 4(d), 4(e), and 4(f), contained some cases with bimodal distribution of HER2 and/or CEP17 and could be categorized as "equivocal with polysomy," Figure 4(d), and "equivocal with polysomy and HER2 bimodality," Figures 4(e) and 4(f) cases. Only one case, Figure 4(e), in the example fits the definition of $\mathrm{GH}$. 

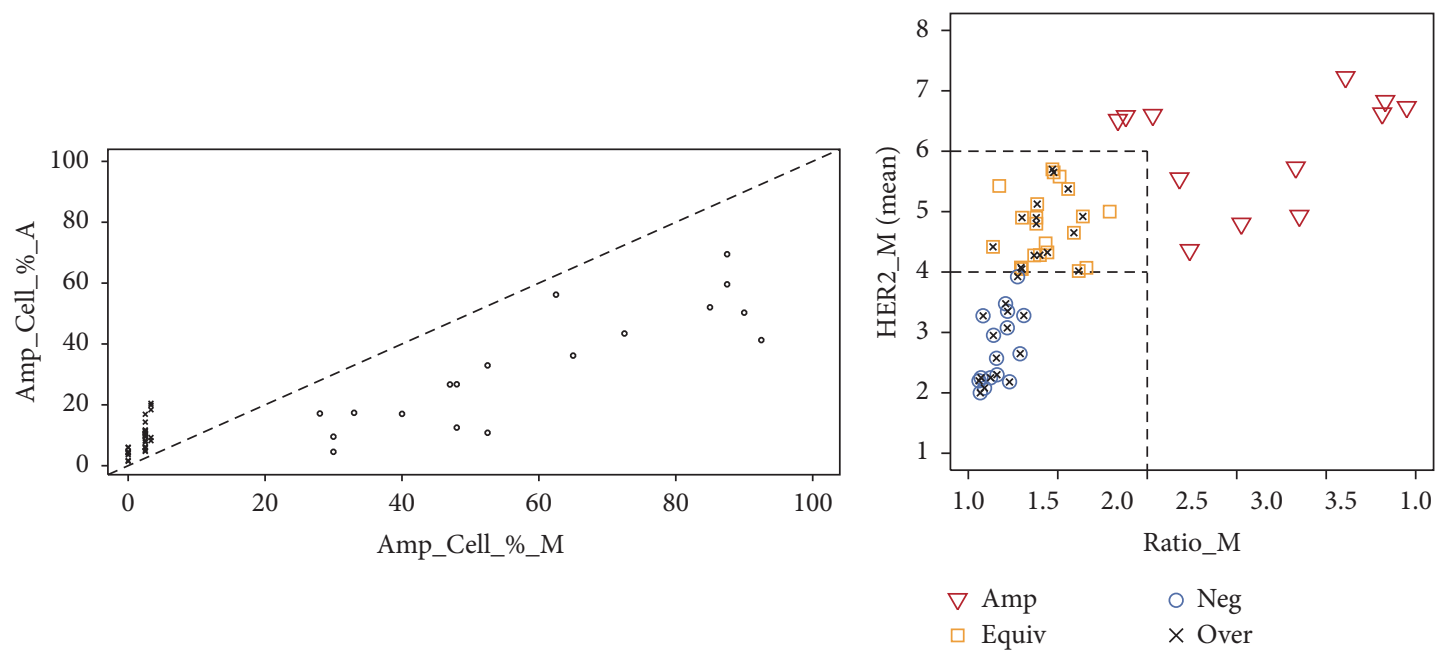

(a)
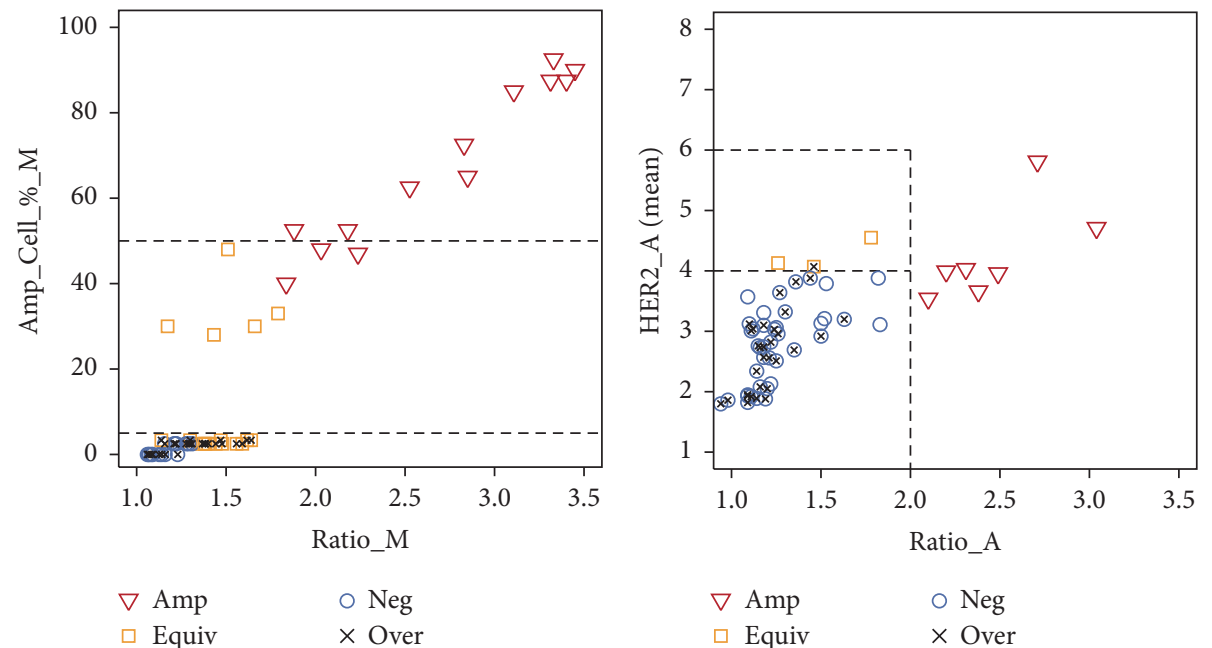

(c)

(d)

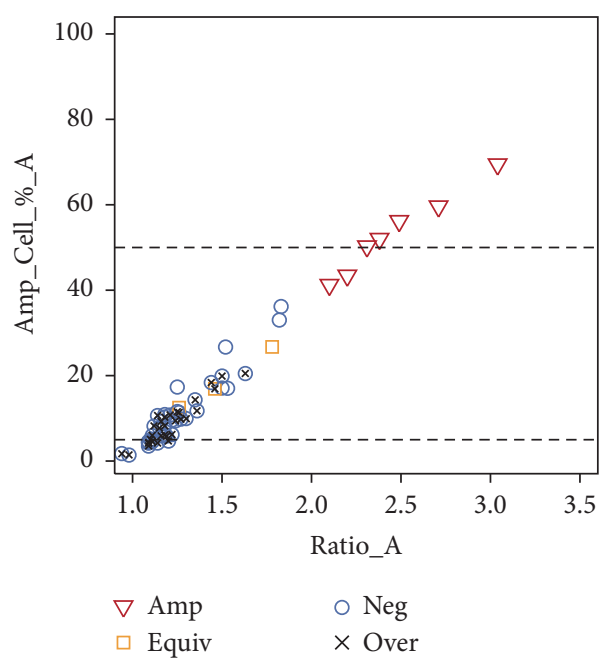

(e)

FIGURE 1: Distribution of tumors with regard to genetic heterogeneity and amplification guidelines. (a) Percentage of amplified cells plotted for MP and AD values, Amp_Cell_\%_M and Amp_Cell_\%_A, respectively. Dashed line marks identity line. AD overestimates low MP values (crosses) whereas it underestimates MP values in the range > 28 (circles); (b) HER2_M plotted against Ratio_M with cut-offs for amplification by ASCO/CAP 2013 guidelines shown by grey lines. Cases marked with crosses are overestimated cases from (a); (c) Cell_Amp_\%_M plotted against Ratio_M, horizontal lines at 5\% and 50\% mark cut-off values for determining GH cases. Note the lack of cases in the 3-28\% range; (d) HER2_A plotted against RATIO_A, amplification cut-off marked in grey; (e) Amp_Cell_\%_A plotted against RATIO_A. Summary: MP: 13 positive, 21 equivocal, 16 negative, and $8 \mathrm{GH}$ cases; AD: 7 positive, 3 equivocal, 40 negative, and $36 \mathrm{GH}$ cases. 


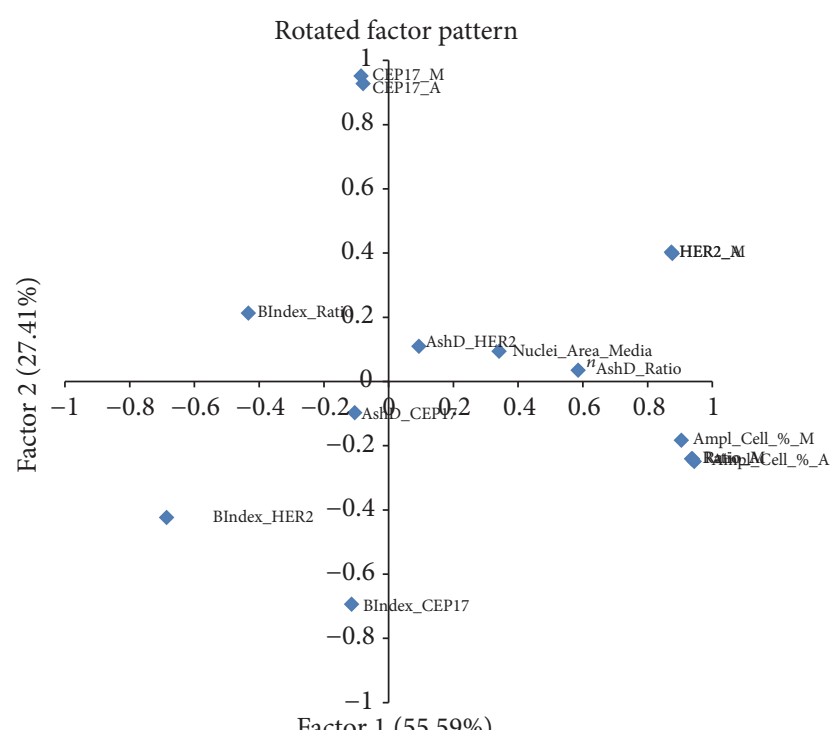

(a)

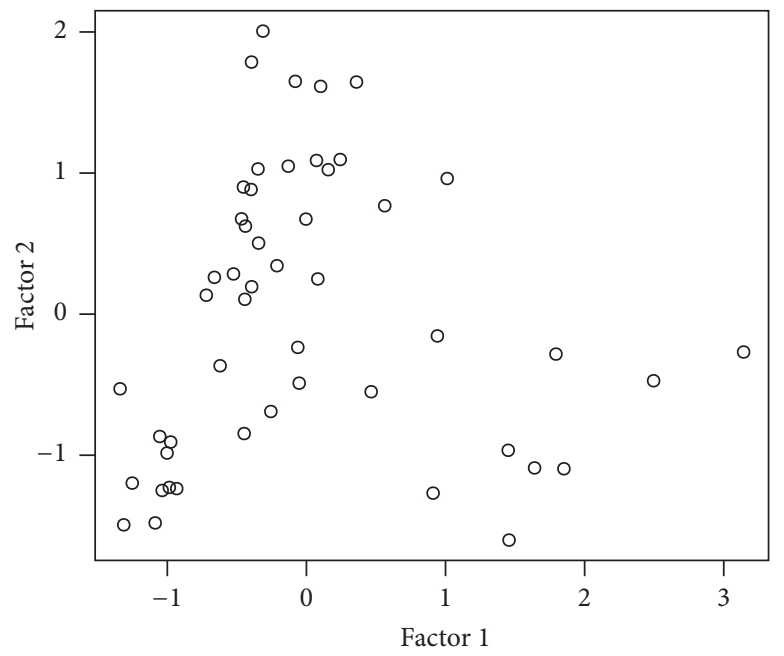

(c)

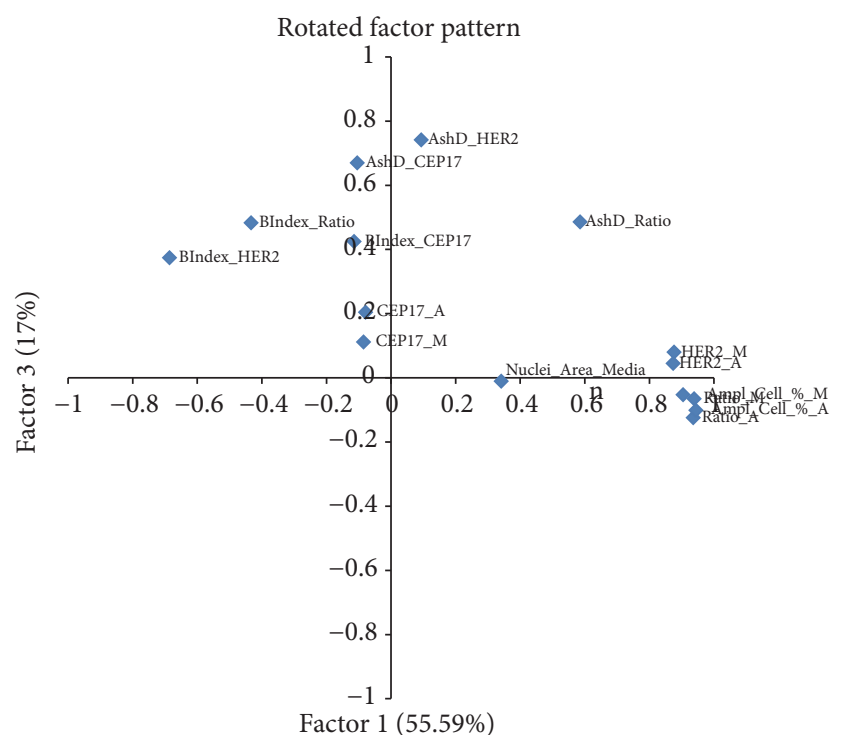

(b)

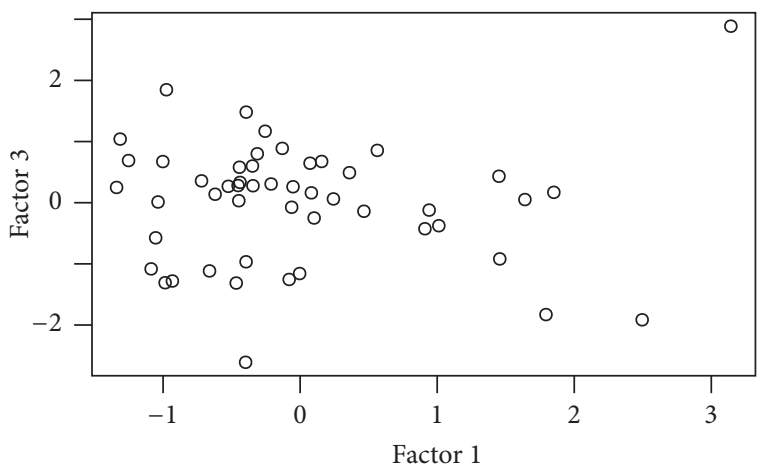

(d)

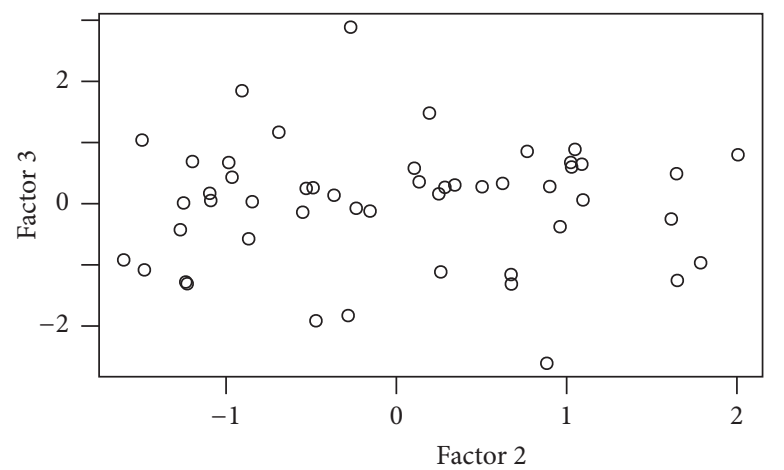

(e)

FIGURE 2: Rotated factor pattern of the indicators obtained by the manual and automated HER2 FISH procedure; $n=50$. The loadings of (a) factors 1 and 2, (b) factors 1 and 3, and the factor scores (c, d, e) are plotted. Factor 1, amplification; factor 2, polysomy; factor 3, bimodality. Cell_Amp_\%_M: percentage of amplified cells detected by manual procedure, calculated from HER2/CEP17 ratio. Cell_Amp_\%_A: percentage of amplified cells detected by automated procedure, calculated from HER2/CEP17 ratio. HER2_A, HER2_M - HER2 copy number detected by automated and manual procedures, respectively. CEP17_A, CEP17_M - CEP17 copy number detected by automated and manual procedures, respectively. Ratio_A, Ratio_M - HER2/CEP17 ratio detected by automated and manual procedures, respectively. AshD_Ratio, AshD_HER2, and AshD_CEP17: Ashman's D indicator calculated for HER2/CEP17, HER2, and CEP17 automated data. BIndex_HER2, BIndex_CEP17, and BIndex_Ratio: bimodality indices calculated for HER2, CEP17, and HER2/CEP17 automated data. 
TABLE 2: Characteristics of the clusters extracted from the automated image analysis data. GH by Cell_Amp_\%_M: percentage of amplified cells detected by manual procedure, calculated from HER2/CEP17 ratio and by HER2 signal only. AshD_Ratio, AshD_HER2, and AshD_CEP17: Ashman's D indicator calculated for HER2/CEP17, HER2, and CEP17 automated data.

\begin{tabular}{|c|c|c|c|c|c|}
\hline & Cluster 1 & Cluster 2 & Cluster 3 & Cluster 4 & Total \\
\hline Number of observations & 1 & 9 & 18 & 22 & 50 \\
\hline $\begin{array}{l}\text { Amplification } \\
\text { (amp/equiv/neg) }\end{array}$ & $1 / 0 / 0$ & $9 / 0 / 0$ & $0 / 4 / 14$ & $3 / 17 / 2$ & 50 \\
\hline Polysomy & 0 & 0 & 3 & 19 & 22 \\
\hline $\begin{array}{l}\text { GH by Cell_Amp_\%_M } \\
\text { (by HER } 2 / C E P 17 \text { ratio) }\end{array}$ & 0 & 1 & 1 & 6 & 8 \\
\hline $\begin{array}{l}\text { GH by Cell_Amp_\%_M } \\
\text { (by HER } 2 \text { only) }\end{array}$ & 0 & 4 & 6 & 17 & 27 \\
\hline AshD_Ratio $>2$ & 1 & 1 & 2 & 1 & 5 \\
\hline AshD_Her2 $>2$ & 1 & 4 & 6 & 12 & 23 \\
\hline AshD_CEP17 > 2 & 1 & 0 & 5 & 5 & 11 \\
\hline Predominantly & Amplified & Amplified & Negative & $\begin{array}{c}\text { Equivocal } \\
\text { Polysomic } \\
\text { Bimodal }\end{array}$ & \\
\hline
\end{tabular}

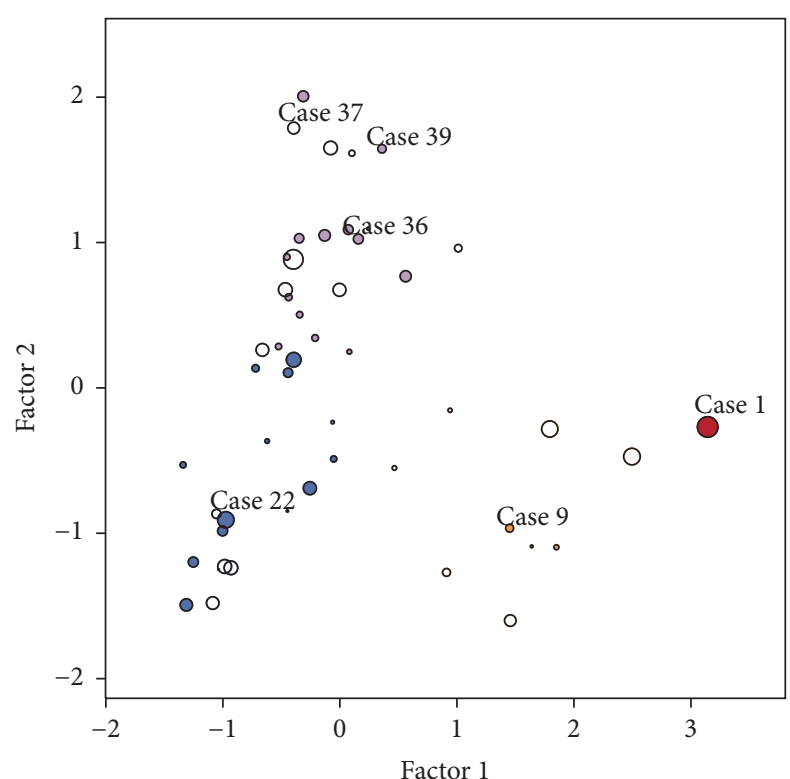

FIGURE 3: A bubble plot of the clusters obtained from the factor 1, 2, and 3 scores. Cluster colors: Cluster 1, red; Cluster 2, orange; Cluster 3, blue; and Cluster 4, purple. Bubble size represents factor 3 (bimodality); center is empty for negative and filled for positive values. Numbers indicate cluster examples depicted in Figure 4.

\section{Discussion}

In our study of 50 HER2 IHC 2+ (borderline) breast carcinomas, we found that automated IA of HER2 FISH images underestimated the conventional assessment by MP and could not readily be used as clinical decision support tool to measure the level of HER2 amplification. However, the benefit of high-capacity nonselective tumor cell assay could be utilized to generate unbiased, quantitative indicators of HER2 intratumor heterogeneity. Importantly, AD revealed a more continuous distribution of the fraction of amplified cells compared to the MP results. Furthermore, AD enabled characteristics of intratumor heterogeneity based on bimodality indicators rather than the fraction of amplified cells. The method also allowed extraction of linearly independent scores of amplification, polysomy, and bimodality with subsequent stratification into relatively unimodal and bimodal tumors; the latter category only partially overlapped with the conventional GH cases.

Clinical HER2 FISH testing is rather straightforward in nonamplified and amplified cases; however, it presents a serious challenge in evaluation of equivocal cases, both by conventional and image analysis-based methods [21, 23, 28]. When therapy decision is to be based on variations within a rather limited number of cells and somewhat arbitrary definitions and cut-off values, robust and evidence-based approaches are needed. Rather than verifying or merely assisting the manual counts of a limited number of cells, high-capacity IA can provide an added value to overcome the limitations of conventional HER2 testing, in particular, by refining the GH concept and highlighting the impact of CEP17 variability on proper interpretation of the test results.

We demonstrate that mathematical bimodality indicators retrieved from the $\mathrm{AD}$ are linearly independent of the level of HER2 amplification or CEP17 polysomy (or HER2/CEP17 ratio). They can therefore serve as objective and quantifiable measures of intratumor heterogeneity, based on true intratumor variation. This is superior to the GH concept which is largely based on the fraction of amplified cells as previously demonstrated by Chang et al. [13] to be dependent on the overall level of amplification. This association was also noted in our study (Figure 1(c)). Bimodality indicators reflect the distribution pattern of the cell population tested and therefore convey different characteristics of the tumor heterogeneity compared to the GH concept. Importantly, we found only partial overlap between GH and bimodal (Ashman's D > 2) cases in our study. Furthermore, in our 

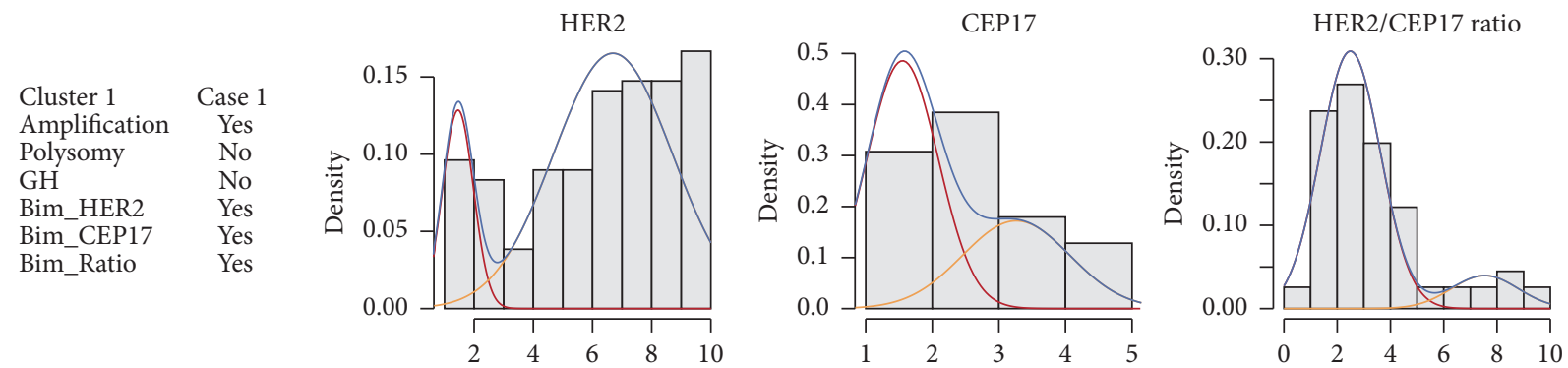

(a)

HER2

Cluster $2 \quad$ Case 9 Amplification Yes Polysomy No GH

Bim HER2

Bim_CEP17

Bim_Ratio

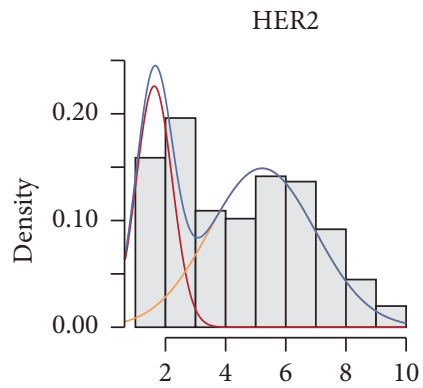

CEP17

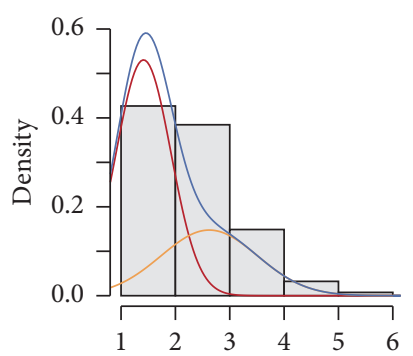

HER2/CEP17 ratio

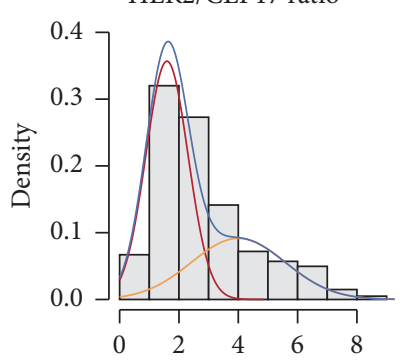

(b)

HER2

CEP17

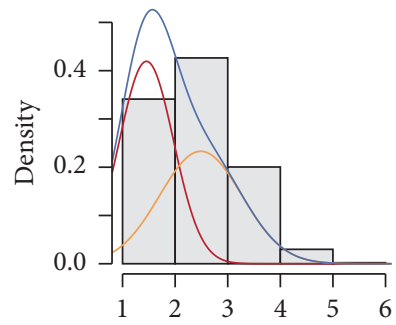

(c)

Case 22

Polysomy No

$\mathrm{GH}$ No

Bim_HER2 Yes

Bim_CEP17 No

Bim_Ratio Yes

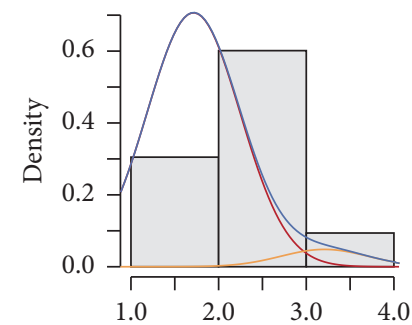

HER2
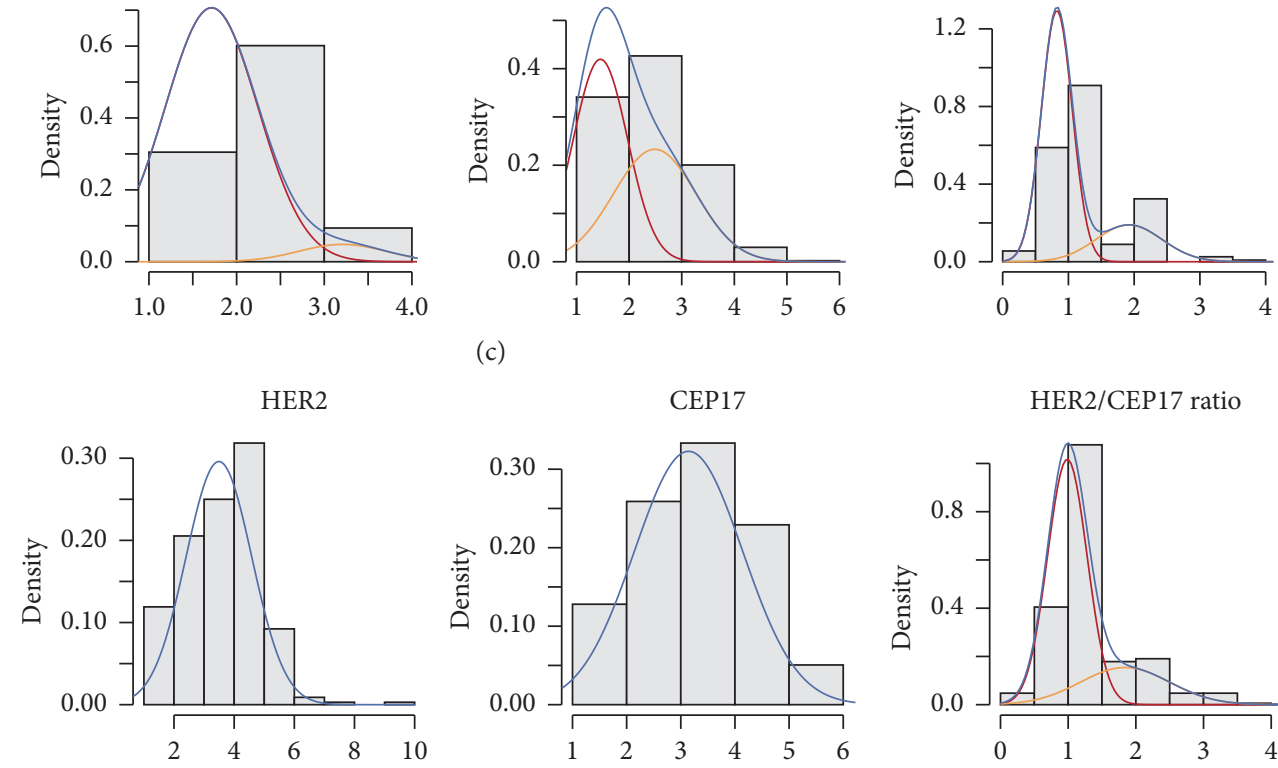

Cluster 4

Case 37

Amplification Equivoca

Polysomy

$\mathrm{GH}$

Bim_HER2

Bim_CEP17

Bim_Ratio

$$
\begin{aligned}
& \text { Yes } \\
& \text { No } \\
& \text { No } \\
& \text { No }
\end{aligned}
$$

(d)

HER2

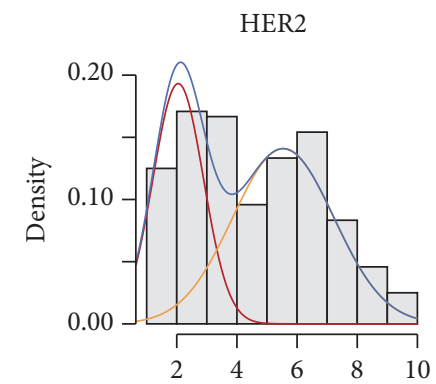

$\begin{array}{lr}\text { Cluster } 4 & \text { Case } 39 \\ \text { Amplification Equivoca }\end{array}$ Amplification Equivoca Polysomy

$\mathrm{GH}$

Yes

Bim_HER2

Bim_CEP17

Bim_Ratio

Yes

Yes

No
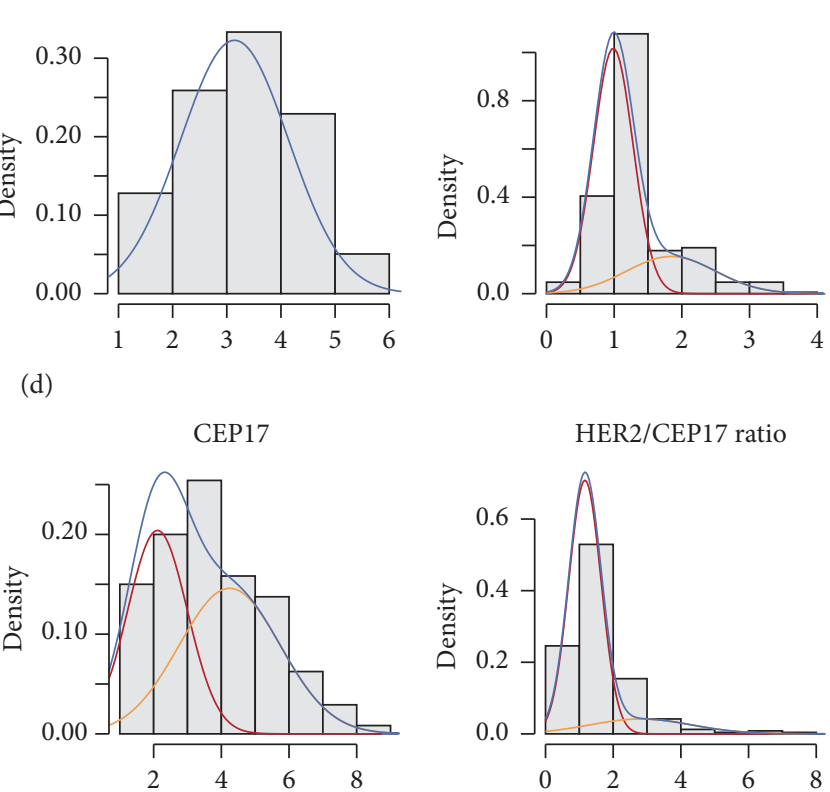

(e)

Figure 4: Continued. 

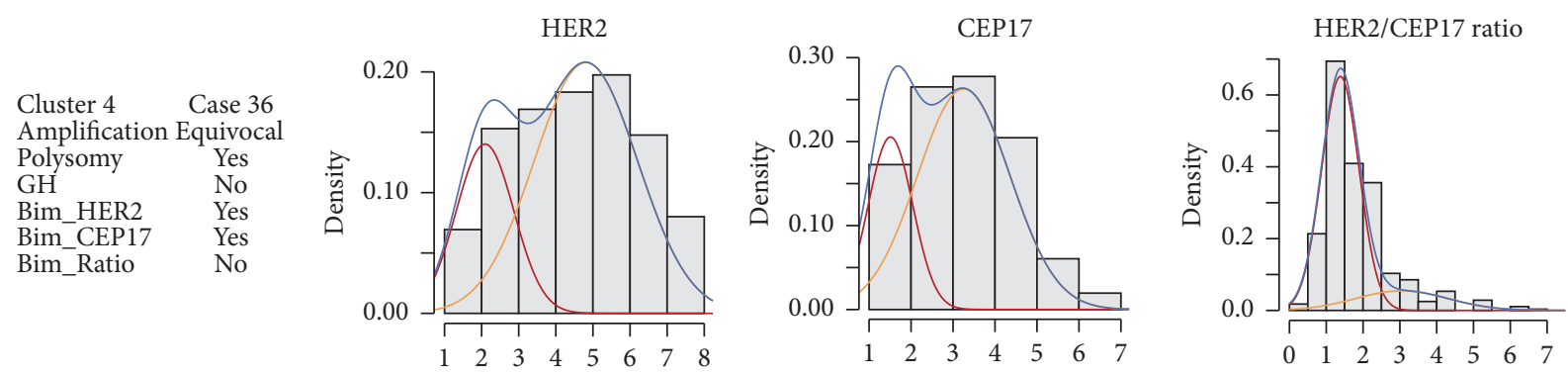

(f)

FIGURE 4: Examples of the tumor cases from the clusters extracted from the automated HER2 FISH data. Histograms of HER2, CEP17, and HER2/CEP17 with Gaussian curves are presented from the cases labeled in the Figure 3. Amplification, polysomy, and genetic heterogeneity categories are based on the conventional manual procedure results. Bim_HER2, Bim_CEP17, and Bim_Ratio represent bimodality categories based on Ashman's D > 2 criterion.

experiments with random simulations of different sample size, we observed that robust detection of the bimodality feature of HER2 amplification in breast cancer tissue requires at least 800 cells. Although larger data sets are needed to establish the cell sampling requirements, they are obviously beyond the MP capacity.

High-capacity automated IA presents another potential benefit for HER2 FISH testing as it sheds the light into distribution of unselected cells and may detect rare amplified cells dispersed in a larger cell population. Comparison of $\mathrm{MP}$ and $\mathrm{AD}$ in our study with regard to the GH definitions revealed peculiar differences in the amplified cell distribution. Amp_Cell_\%_A and Amp_Cell_\%_M plots (Figure 1) exposed a large distribution gap in the MP data. Namely, all GH cases detected by MP contained at least $28 \%$ amplified cells. On the other hand, out of 32 cases with Amp_Cell_\%_M under 5\%, 24 cases disclosed 5 to $21 \%$ by Amp_Cell_\%_A (there were no cases detected by MP in the interval from 5 to $21 \%$ ). There were 9 cases with less than $5 \%$ amplified cells detected automatically. This discrepancy might be associated with scattered amplified cells which could be missed by MP as it requires tedious screening of large tissue area. To support this hypothesis, we found that the median distance between the amplified nuclei was significantly higher in the cases undisclosed by MP. It points to the potential of IA to capture the cell amplification/heterogeneity to full extent. The range of the gap fits well with reports from other studies investigating the amplification cut-off values in the GH guidelines: the questionability of the lower limit (5\%) of the amplification threshold was argued by a possible misclassification of the case as heterogeneous when only 1 cell with a HER2/CEP17 ratio $>2.2$ in 20 is counted $[12,16,18]$. Layfield and Schmidt showed that the cells with 3:1 HER2/CEP17 ratio were the determining factor for $\mathrm{GH}$ in $46 \%$ of heterogeneous cases, while $35 \%$ of $\mathrm{GH}$ cases were established due to a single $3: 1$ cell [16]. It is known that loss of CEP17 signal may be the result of nuclear truncation [29]. On the other hand, Bartlett et al. reported that only cases containing $>30 \%$ of amplified cells (HER2/CEP17 ratios more than 2.2) were associated with lower disease-free survival [12]. Allison et al. showed that different amplification ranges dichotomize cohorts very differently: nonamplified and heterogeneous cases accumulated in the $5 \%$ to $15 \%$ range, while equivocal and heterogeneous gathered in the $25 \%$ to $35 \%$ interval [18]. They suggested that the recommended thresholds used for reporting heterogeneity may be too low.

Our study is limited by the lack of outcome data to test the clinical/predictive value of the bimodality indicators for evaluation of HER2 amplification intratumor heterogeneity. Neither was it our goal to calibrate a computer-assisted tool to count FISH signals in a limited number of cells according to the current clinical guidelines. Previous studies on HER2 FISH image analysis to support evaluation of HER2 status did not explore the advantages of larger cell sampling and were usually based on 40-60 nuclei [22, 23], or "at least 60 valid nuclei" [21], or "an average of 113 cells per case (median 94, range 47-254)" [25]. Our results suggest that automated image analysis can add value by retrieving new quality information on intratumor heterogeneity rather than merely supporting manual evaluation according to the current clinical guidelines. Indeed, bimodality indicators are more mathematically appropriate and may prove to be more biologically relevant features of intratumor heterogeneity to be considered in clinical settings and definitions of $\mathrm{GH}$. Of note, bimodality indicators of Ki67 IHC data have been recently shown to be an independent prognostic factor of overall survival in breast cancer patients; remarkably, the bimodality of intratumor distribution of Ki67 positive tumor cells was more powerful prognostic factor than the Ki67 labeling index per se [30].

In conclusion, the automated HER2 FISH image analysis in our study underestimated the HER2 and CEP17 data obtained by conventional HER2 FISH test. This bias is most likely caused by cell selection differences in the manual and automated procedures and makes the image analysis not readily applicable according to the current clinical guidelines. However, a unique benefit of the automated high-capacity nonselective tumor cell assay can be obtained from generating unbiased, quantitative indicators of HER2 intratumor heterogeneity with regard to HER2, CEP17 signals, and their ratios. The method also allowed extraction of linearly independent scores of amplification, polysomy, and bimodality with subsequent stratification into relatively unimodal and bimodal tumors, with only partial overlap to the conventional 
GH cases. Importantly, IA revealed a continuous distribution of amplified cells without gaps which improved detection of rare amplified cells in the tumors with less than 5\% amplified cells by conventional HER2 FISH test.

\section{Conflicts of Interest}

The authors declare that they have no conflicts of interest.

\section{Acknowledgments}

The authors acknowledge financial support of the EC under the Marie Curie Industry-Academia Partnership and Pathways (Project "Academia-Industry Collaboration for Digital Pathology”; Grant Agreement no. 612471).

\section{References}

[1] D. J. Slamon, G. M. Clark, S. G. Wong, W. J. Levin, A. Ullrich, and W. L. McGuire, "Human breast cancer: correlation of relapse and survival with amplification of the HER-2/neu oncogene," Science, vol. 235, no. 4785, pp. 177-182, 1987.

[2] C. Gutierrez and R. Schiff, "HER2: biology, detection, and clinical implications," Archives of Pathology \& Laboratory Medicine, vol. 135, no. 1, pp. 55-62, 2011.

[3] A. C. Wolff, M. E. Hammond, and D. G. Hicks, "Recommendations for human epidermal growth factor receptor 2 testing in breast cancer: American Society of Clinical Oncology/College of American Pathologists clinical practice guideline update," Journal Clinical Oncology, vol. 31, no. 31, pp. 3997-4013, 2013.

[4] D. G. Hicks and S. Kulkarni, "Trastuzumab as adjuvant therapy for early breast cancer: the importance of accurate human epidermal growth factor receptor 2 testing," Archives of Pathology \& Laboratory Medicine, vol. 132, no. 6, pp. 1008-1015, 2008.

[5] J. Krell, C. R. James, D. Shah et al., "Human epidermal growth factor receptor 2-positive breast cancer relapsing post-adjuvant trastuzumab: pattern of recurrence, treatment and outcome," Clinical Breast Cancer, vol. 11, no. 3, pp. 153-160, 2011.

[6] A. C. Wolff, M. E. H. Hammond, J. N. Schwartz et al., "American Society of Clinical Oncology/College of American Pathologists guideline recommendations for human epidermal growth factor receptor 2 testing in breast cancer," Journal of Clinical Oncology, vol. 25, no. 1, pp. 118-145, 2007.

[7] B. Bernasconi, A. M. Chiaravalli, G. Finzi, K. Milani, and M. G. Tibiletti, "Genetic heterogeneity in HER2 testing may influence therapy eligibility," Breast Cancer Research and Treatment, vol. 133, no. 1, pp. 161-168, 2012.

[8] A. Sapino, M. Goia, D. Recupero, and C. Marchiò, "Current challenges for HER2 testing in diagnostic pathology: state of the art and controversial issues," Frontiers in Oncology, vol. 3, article 129, 2013.

[9] C. Marchiò, M. B. Lambros, P. Gugliotta et al., "Does chromosome 17 centromere copy number predict polysomy in breast cancer? A fluorescence in situ hybridization and microarraybased CGH analysis," Journal of Pathology, vol. 219, no. 1, pp. 16-24, 2009.

[10] I.-T. Yeh, M. A. Martin, R. S. Robetorye et al., "Clinical validation of an array CGH test for HER2 status in breast cancer reveals that polysomy 17 is a rare event," Modern Pathology, vol. 22, no. 9, pp. 1169-1175, 2009.
[11] W. M. Hanna, J. Rüschoff, M. Bilous et al., "HER2 in situ hybridization in breast cancer: clinical implications of polysomy 17 and genetic heterogeneity," Modern Pathology, vol. 27, no. 1, pp. 4-18, 2014.

[12] A. I. Bartlett, J. Starcyznski, T. Robson et al., "Heterogeneous HER2 gene amplification: impact on patient outcome and a clinically relevant definition," American Journal of Clinical Pathology, vol. 136, no. 2, pp. 266-274, 2011.

[13] M. C. Chang, J. I. Malowany, J. Mazurkiewicz, and M. Wood, “'Genetic heterogeneity' in HER2/neu testing by fluorescence in situ hybridization: a study of 2522 cases," Modern Pathology, vol. 25, no. 5, pp. 683-688, 2012.

[14] G. H. Vance, T. S. Barry, K. J. Bloom et al., "Genetic heterogeneity in HER2 testing in breast cancer: panel summary and guidelines," Archives of Pathology \& Laboratory Medicine, vol. 133, no. 4, pp. 611-612, 2009.

[15] H. Seol, H. J. Lee, Y. Choi et al., "Intratumoral heterogeneity of HER2 gene amplification in breast cancer: its clinicopathological significance," Modern Pathology, vol. 25, no. 7, pp. 938-948, 2012.

[16] L. J. Layfield and R. L. Schmidt, "HER2/neu gene amplification heterogeneity: the significance of cells with a 3:1 HER2/CEP17 ratio," Applied Immunohistochemistry and Molecular Morphology, vol. 20, no. 6, pp. 543-549, 2012.

[17] C. Y. Hsu, A. F. Li, C. F. Yang, and D. M. Ho, "Proposal of modification for the definition of genetic heterogeneity in HER2 testing in breast cancer," Archives of Pathology \& Laboratory Medicine, vol. 134, no. 2, pp. 162-163, 2010.

[18] K. H. Allison, S. M. Dintzis, and R. A. Schmidt, "Frequency of HER2 heterogeneity by fluorescence in situ hybridization according to CAP expert panel recommendations: time for a new look at how to report heterogeneity," American Journal of Clinical Pathology, vol. 136, no. 6, pp. 864-871, 2011.

[19] C. Öhlschlegel, K. Zahel, D. Kradolfer, M. Hell, and W. Jochum, "HER2 genetic heterogeneity in breast carcinoma," Journal of Clinical Pathology, vol. 64, no. 12, pp. 1112-1116, 2011.

[20] C. López, B. Tomás, A. Korzynska et al., "Is it necessary to evaluate nuclei in HER2 FISH evaluation?" American Journal of Clinical Pathology, vol. 139, no. 1, pp. 47-54, 2013.

[21] Z. Theodosiou, I. N. Kasampalidis, G. Karayannopoulou et al., "Evaluation of FISH image analysis system on assessing HER2 amplification in breast carcinoma cases," Breast, vol. 17, no. 1, pp. 80-84, 2008.

[22] M. J. D. Prins, J. P. Ruurda, P. J. van Diest, R. van Hillegersberg, and F. J. W. ten Kate, "Evaluation of the HER2 amplification status in oesophageal adenocarcinoma by conventional and automated FISH: a tissue microarray study," Journal of Clinical Pathology, vol. 67, no. 1, pp. 26-32, 2014.

[23] D. Furrer, S. Jacob, C. Caron, F. Sanschagrin, L. Provencher, and C. Diorio, "Validation of a new classifier for the automated analysis of the human epidermal growth factor receptor 2 (HER2) gene amplification in breast cancer specimens," Diagnostic Pathology, vol. 8, no. 1, article 17, 2013.

[24] B. Reljin, M. Paskas, I. Reljin, and K. Konstanty, "Breast cancer evaluation by fluorescent dot detection using combined mathematical morphology and multifractal techniques," Diagnostic Pathology, vol. 6, supplement 1, p. S21, 2011.

[25] J. Konsti, J. Lundin, M. Jumppanen, M. Lundin, A. Viitanen, and J. Isola, "A public-domain image processing tool for automated quantification of fluorescence in situ hybridisation signals," Journal of Clinical Pathology, vol. 61, no. 3, pp. 278-282, 2008. 
[26] G. Pajor, B. Kajtár, L. Pajor, and D. Alpár, "State-of-the-art FISHing: automated analysis of cytogenetic aberrations in interphase nuclei," Cytometry Part A, vol. 81, no. 8, pp. 649-663, 2012.

[27] M. Salido, I. Tusquets, J. M. Corominas et al., "Polysomy of chromosome 17 in breast cancer tumors showing an overexpression of ERBB2: a study of 175 cases using fluorescence in situ hybridization and immunohistochemistry," Breast Cancer Research, vol. 7, no. 2, pp. R267-R273, 2005.

[28] R. Stevens, I. Almanaseer, M. Gonzalez et al., "Analysis of HER2 gene amplification using an automated fluorescence in situ hybridization signal enumeration system," Journal of Molecular Diagnostics, vol. 9, no. 2, pp. 144-150, 2007.

[29] E. Kouvaras, C. N. Papandreou, D. D. Daliani, A. Athanasiadis, and G. K. Koukoulis, "Comparative study of spatial localization of HER-2 and CEP17 signals and of HER-2/CEP17 ratios, in 'thin' and 'thick' tissue sections," Breast, vol. 21, no. 1, pp. 3439, 2012.

[30] A. Laurinavicius, B. Plancoulaine, A. Rasmusson et al., "Bimodality of intratumor Ki67 expression is an independent prognostic factor of overall survival in patients with invasive breast carcinoma," Virchows Archiv, vol. 468, no. 4, pp. 493-502, 2016. 


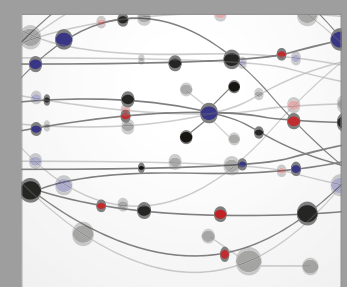

The Scientific World Journal
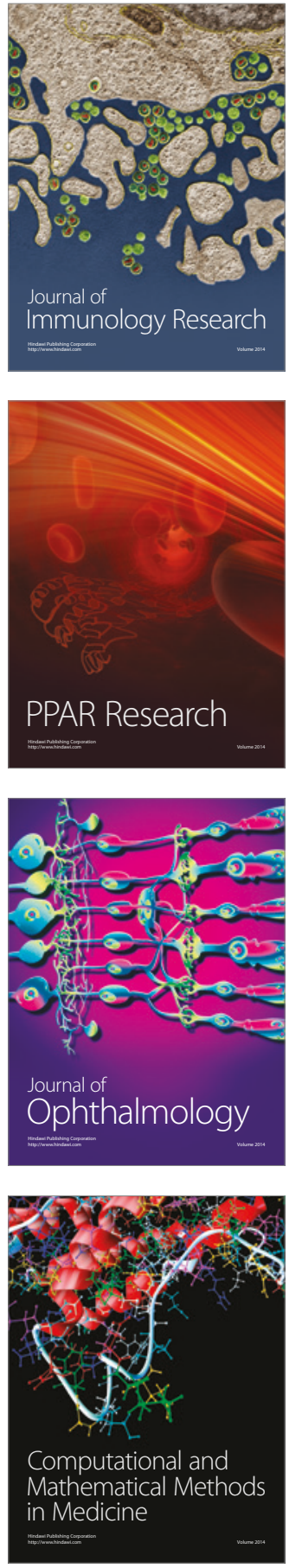

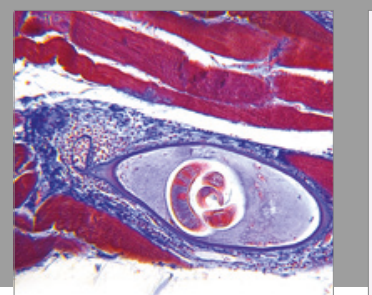

Gastroenterology Research and Practice
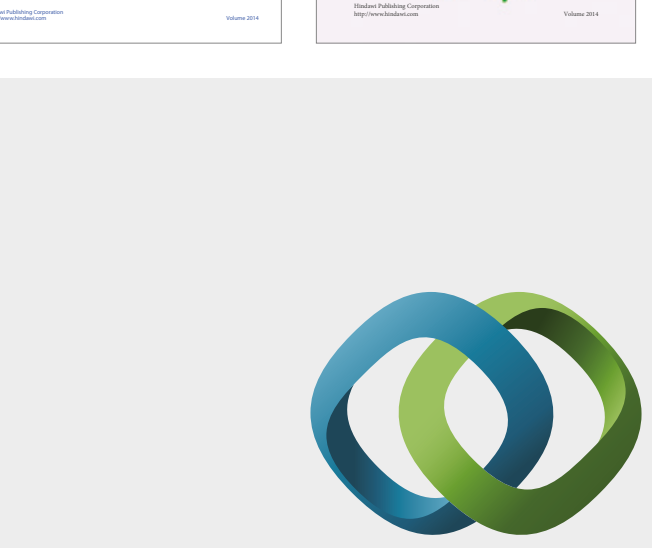

\section{Hindawi}

Submit your manuscripts at

https://www.hindawi.com
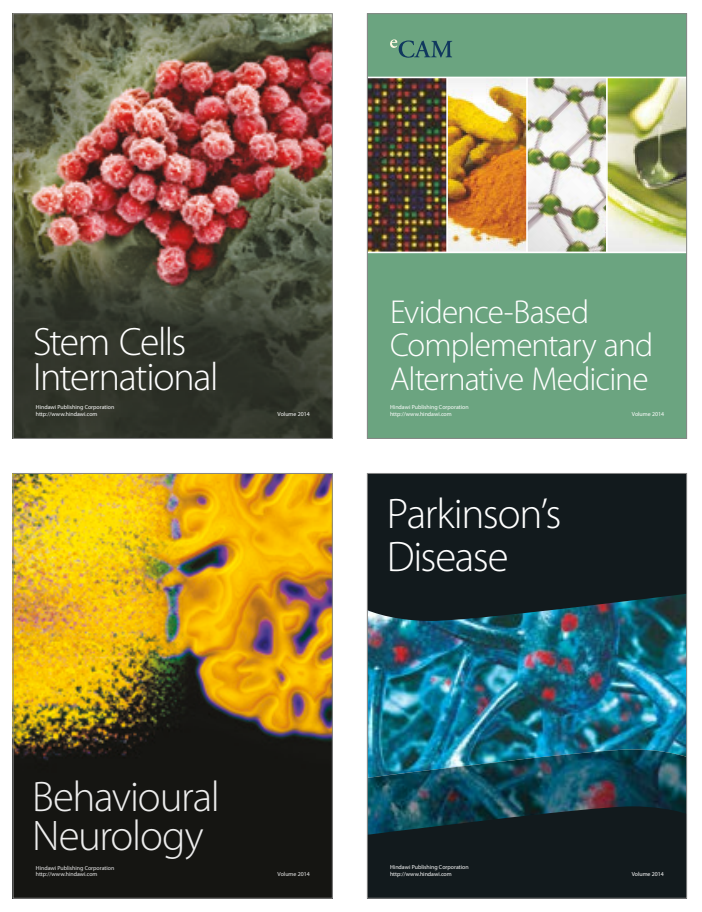
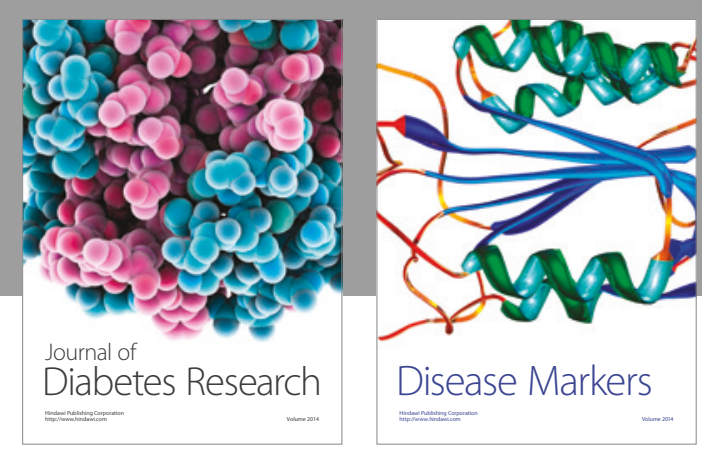

Disease Markers
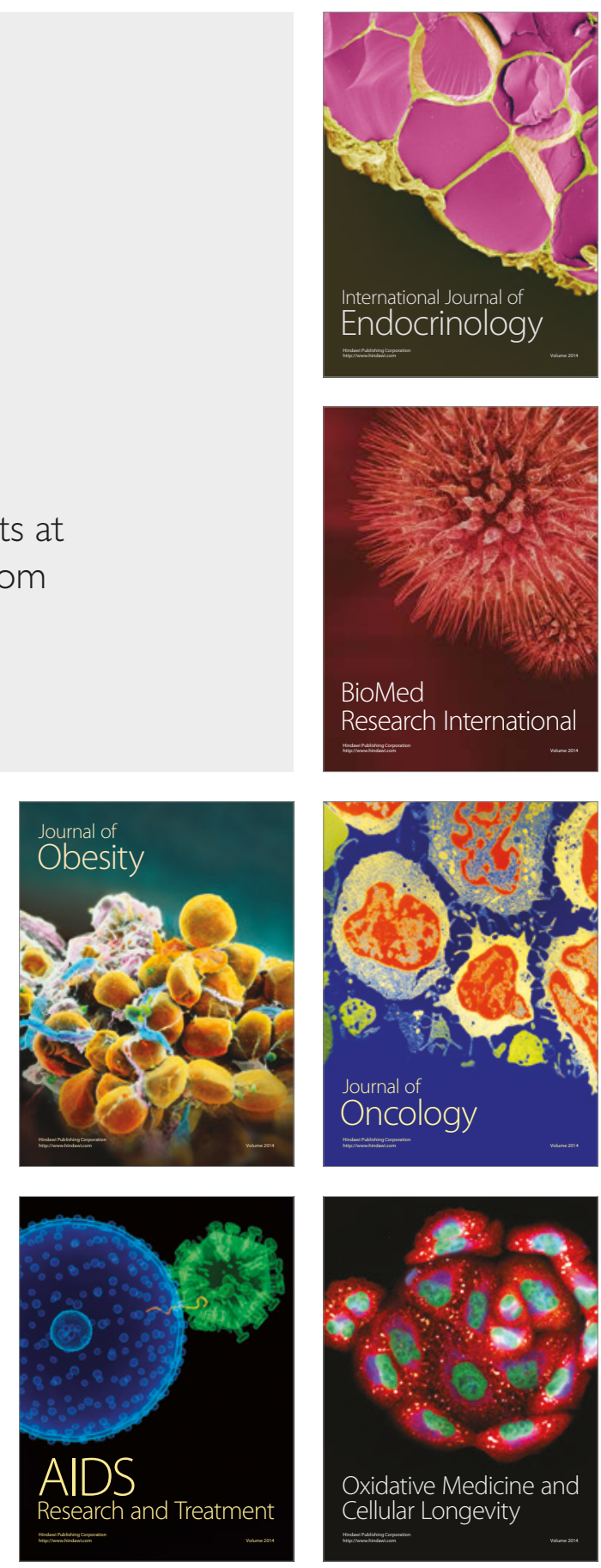